\title{
Short-term outcome of lamina-sparing decompression in thoracolumbar spinal tuberculosis
}

\author{
Gagandeep Yadav, MS, Pankaj Kandwal, MS, and Shobha S. Arora, MS \\ Department of Orthopaedics, All India Institute of Medical Sciences, Rishikesh, Uttarakhand, India
}

\begin{abstract}
OBJECTIVE The authors sought to assess the outcomes of lamina-sparing decompression using a posterior-only approach in patients with thoracolumbar spinal tuberculosis (TB). In patients with spinal TB with paraplegia, anterior decompression yields excellent results because it allows direct access to the diseased part of the vertebra, but the anterior approach has related morbidities. Posterior and posterolateral decompression mitigate approach-related morbidities; however, these approaches destabilize the already diseased segment. Lamina-sparing decompression through a posterior-only approach is a modification of posterolateral and anterolateral decompression that allows simultaneous decompression and instrumentation while preserving the posterior healthy bony structure as much as possible.
\end{abstract}

METHODS Thirty-five patients with spinal TB underwent lamina-sparing decompression and instrumentation. Outcomes were determined by using a visual analog scale (VAS) and the Oswestry Disability Index (ODI) for functional assessment, the American Spinal Injury Association (ASIA) impairment grade for neurological assessment, blood loss and duration of surgery for surgical outcome assessment, and Cobb angles to measure kyphosis correction.

RESULTS In total, 35 patients (12 men and 23 women) with an average age of $35.8 \pm 18.7$ (range 4-69) years underwent lamina-sparing decompression. Eight patients had dorsal, 7 had dorsolumbar, 7 had lumbar, 9 had multifocal contiguous, and 4 patients had multifocal noncontiguous spinal TB; 33 patients had paradiscal Pott's spine (tuberculous spondylodiscitis), and 2 had central-type disease. The average preoperative Cobb angle was $28.4^{\circ} \pm 14.9^{\circ}$ (range $\left.0^{\circ}-60^{\circ}\right)$ and the postoperative Cobb angle was $16.3^{\circ} \pm 11.3^{\circ}\left(44^{\circ}\right.$ to $\left.-15^{\circ}\right)$. There was loss of $1.6^{\circ} \pm 1.5^{\circ}\left(0^{\circ}-5^{\circ}\right)$ during 16 months of follow-up. Average blood loss was $526 \pm 316$ (range 130-1200) ml. Duration of surgery was $228 \pm 79.14$ (range 60-320) minutes. Level of vertebral instrumentation on average was $0.97 \pm 0.8$ (range $0-4$ ) vertebra proximal and $1.25 \pm 0.75(0-3)$ distal to the diseased segment. Neurological recovery during the immediate postoperative period occurred in 23 of 27 patients (85.1\%). All patients had recovered at the final follow-up at 16 months. The preoperative ODI score improved from $76.4 \pm 17.9$ (range 32-100) to $6.74 \pm 17.2(0-60)$ at 16 months. The preoperative VAS score improved from $7.48 \pm 1.16(6-10)$ to $0.47 \pm 1.94(0-8)$. Surgical site infection occurred in 2 patients, and 1 patient had an intraoperative dural tear that was successfully repaired. One patient developed implant loosening at 3 months, which was managed by extended instrumentation.

CONCLUSIONS To achieve stability, lamina-sparing decompression allows fixation of lower numbers of vertebrae proximal and distal to the diseased segment. This method has a fair outcome in terms of kyphosis correction, good functional and neurological recovery, shorter surgical duration than conventional methods, and less blood loss.

https://thejns.org/doi/abs/10.3171/2020.1.SPINE191152

KEYWORDS Pott's spine; posterolateral decompression; anterolateral decompression; short instrumentation; anterior column reconstruction; $360^{\circ}$ fusion; thoracic; lumbar; infection; surgical technique

$\mathrm{S}$ PINAL tuberculosis (TB) accounts for $50 \%$ of musculoskeletal TB, ${ }^{1}$ and the incidence of neurological complications varies from $10 \%$ to $43 \%$. $^{1,2}$ Spinal TB is associated with significant morbidity and mortality. ${ }^{3}$ The incidence of TB spine is increasing in both developed and developing countries, making it a major public health concern. ${ }^{2}$ Although antitubercular treatment (ATT) is the mainstay of disease control, surgery is indicated for patients with paraplegia, persistent or progressive neurological deficits despite being on ATT, multifocal contiguous TB spine, pan-vertebral disease, instability, spinal tumor syndrome, deformity correction, drainage of pre- or para-

ABBREVIATIONS ASIA = American Spinal Injury Association; ATT = antitubercular treatment; ODI = Oswestry Disability Index; TB = tuberculosis; VAS = visual analog scale.

SUBMITTED September 23, 2019. ACCEPTED January 13, 2020.

INCLUDE WHEN CITING Published online March 20, 2020; DOI: 10.3171/2020.1.SPINE191152. 
vertebral abscess, neural arch disease, ${ }^{2}$ or severe or persisting instability and pain despite being on ATT, and in children younger than 10 years who have "spine at risk" signs. ${ }^{4}$ The conventional approach for TB spine surgery has been the anterior approach because it provides direct access to diseased vertebrae, ${ }^{5}$ but over time the trend has shifted toward a posterior approach because of the high morbidity related to the anterior approach. Furthermore, the posterior elements are usually relatively healthier in TB spine, which provides better implant purchase with posterior instrumentation. ${ }^{6}$ However, the posterior approach destabilizes the spine during the course of decompression due to removal of healthy posterior elements, requiring longer instrumentation to achieve stability and thus compromising mobility at adjacent healthy instrumented segments. This process has a more pronounced effect at the lumbar spine, which is an important region for spine mobility in the sagittal and coronal planes and rotation. Longer instrumentation also adds extra cost to healthcare. Hence, we devised an approach which allows removal of as minimal an amount of posterior elements as possible, and we named it "lamina-sparing decompression." This approach allows simultaneous decompression and instrumentation without cord handling, while preserving the anatomy of the vertebrae. In this study, we assessed surgical, functional, radiological, and neurological outcomes of lamina-sparing decompression in patients with thoracolumbar spine TB.

\section{Methods}

This investigation was conducted as a prospective observational cohort study at the All India Institute of Medical Sciences from December 2016 to January 2019. Ethical clearance was obtained from the ethics committee of our institution. In total, 206 patients presented to our TB spine clinic during this time period, of whom 166 were managed conservatively and 40 were managed surgically. All patients who required surgical intervention were informed about the study. Willing patients were enrolled after providing written informed consent.

Patients with active thoracolumbar spine TB with mechanical instability of the spine or progressive/persistent profound neural deficits despite being on 4 weeks of ATT were included in the study. Patients with healed spine TB, spinal tumor syndrome, or neural arch disease were excluded from the study. Out of 40 patients, 2 patients had cervicodorsal junction TB and 2 met exclusion criteria; hence, 36 patients were enrolled in the study. One patient was lost to follow-up, and hence the final analysis was done in 35 patients.

The various outcomes measured were blood loss and duration of surgery, American Spinal Injury Association (ASIA) grade for neurological assessment, visual analog scale (VAS) and Oswestry Disability Index (ODI) scores for functional improvement/deterioration, and Cobb angle for radiological assessment. The follow-up range was 12 24 months, with an average follow-up of 16 months.

\section{Surgical Technique}

The anesthetized patient was placed in a prone position,

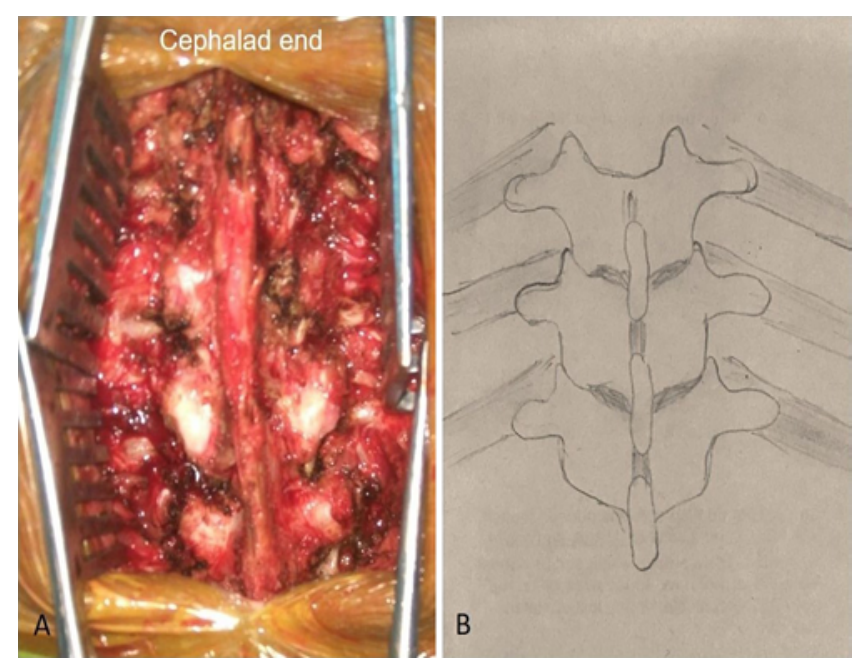

FIG. 1. Exposure of posterior elements. Intraoperative photograph (A) and illustration of the same (B). Figure is available in color online only.

and a posterior midline incision was made over the diseased segment. The paraspinal muscles were elevated subperiosteally and retracted (Fig. 1). Pedicle screws were inserted into proximal and distal healthy adjacent vertebrae. Stabilization on the opposite side of the vertebrae was done with the help of a temporary rod (Fig. 2). Resection of at least 5-7 cm of the medial part of the rib, including the transverse process, costotransverse joint, costovertebral joint, and pedicle, was done, and cord decompression was performed (Fig. 3). Reconstruction of the anterior column was done with either a cage or a graft. Posterior and posterolateral grafting were done, and stabilization was obtained with a contoured rod (Fig. 4). The wound was closed in layers. ATT was resumed from the morning of the 1st postoperative day. All patients were mobilized with rigid external orthosis on the 3rd or 4th postoperative day. Walking was allowed depending on neurological status. ATT was continued as long as contrast-enhanced MRI showed persistent disease activity and erythrocyte sedimentation rate (ESR) and high-sensitivity C-reactive protein levels (CRPH) were raised.

Statistical analysis was performed with IBM SPSS Statistics version 1.0.0-3112. Paired t-tests were applied for pre- and postoperative analysis of ODI and VAS scores and kyphosis correction.

\section{Results}

In the 35 study patients ( 12 men and 23 women, average age $35.8 \pm 18.7$ years, range $4-69$ years), 8 patients had dorsal, 7 had dorsolumbar, 7 had lumbar, 9 had multifocal contiguous, and 4 patients had multifocal noncontiguous spinal TB; 33 patients had paradiscal Pott's spine (tuberculous spondylodiscitis) and 2 had central-type disease. The average preoperative Cobb angle was $28.4^{\circ} \pm 14.9^{\circ}$ (range $0^{\circ}-60^{\circ}$ ), and the postoperative Cobb angle was $16.3^{\circ} \pm 11.3^{\circ}\left(44^{\circ}\right.$ to $\left.-15^{\circ}\right)$. Average kyphosis correction was $18.2^{\circ} \pm 12 \cdot 7^{\circ}\left(0^{\circ}-40^{\circ}\right)$. Kyphosis at the final follow-up of 16 months was $19.4^{\circ} \pm 11.8^{\circ}\left(0^{\circ}-44^{\circ}\right)$. There was loss of $1.6^{\circ} \pm 1.5^{\circ}\left(0^{\circ}-5^{\circ}\right)$ during the 16 -month follow-up. There 


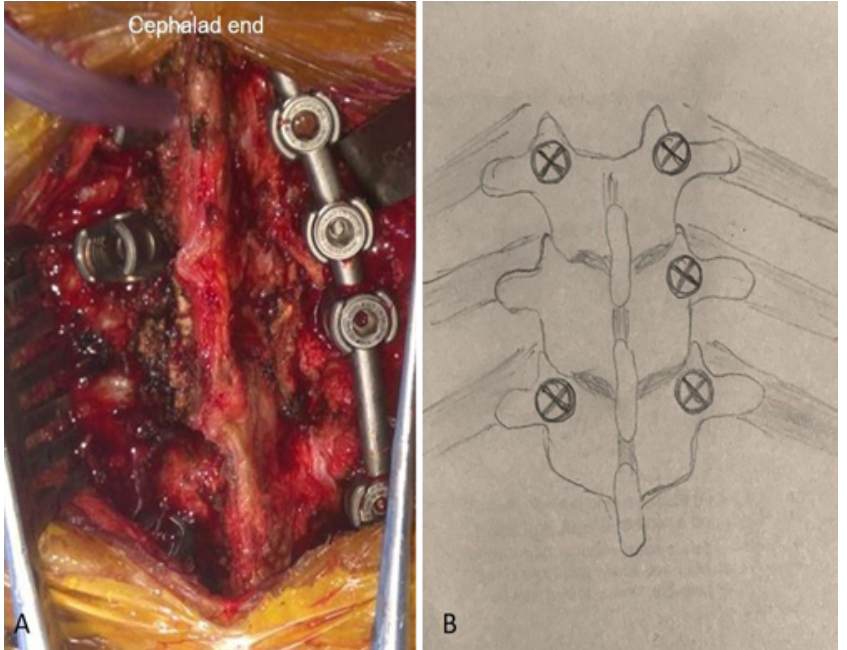

FIG. 2. Exposure of vertebral stabilization with a temporary rod. Intraoperative photograph (A) and illustration (B). Figure is available in color online only.

was a statistically significant difference $(p<0.5)$ between preoperative and postoperative kyphosis and preoperative and final kyphosis at 16 months, suggesting that statistically significant correction was achieved. The difference between the postoperative and final kyphosis angle at 16 months was not statistically significant, suggesting that loss of kyphosis was minimal. The average blood loss during surgery was $526 \pm 316$ (range 130-1200) ml. Duration of surgery was $228 \pm 79.14$ (range 60-320) minutes. The average numbers of diseased vertebrae were $2.8 \pm 1.4$ (range 1-6) and of instrumented vertebra were $3.77 \pm 1.6$ $(0-7)$. The level of instrumentation on average was $0.97 \pm$
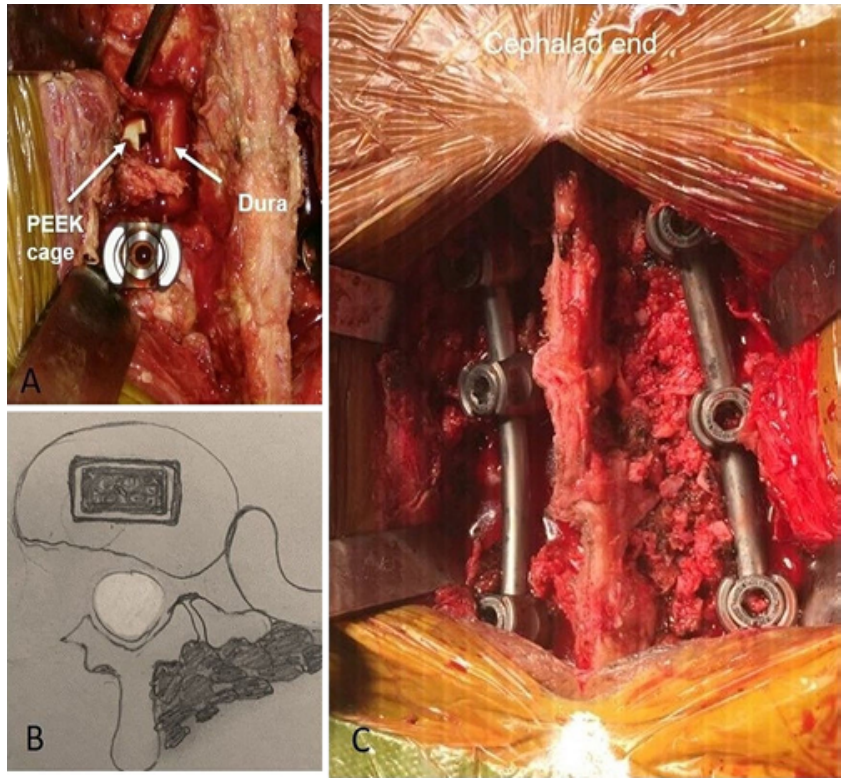

FIG. 4. Cage insertion. Intraoperative photograph (A), illustration of axial section at the level of the cage and bone grafting $(B)$, and intraoperative photograph showing posterior and posterolateral bone grafting for fusion (C). Figure is available in color online only.

0.8 (range 0-4) vertebra proximal and $1.25 \pm 0.75(0-3)$ vertebra distal to the diseased segment. In our cohort of 35 patients, 8 did not have any neurological deficits, while 27 patients had neurological deficit. Twenty-three out of 27 $(85.1 \%)$ patients had neurological recovery during the immediate postoperative period. All patients had recovered $(100 \%)$ by a few ASIA grades at the final follow-up of 16 months. None of the 8 patients with intact neurologi-

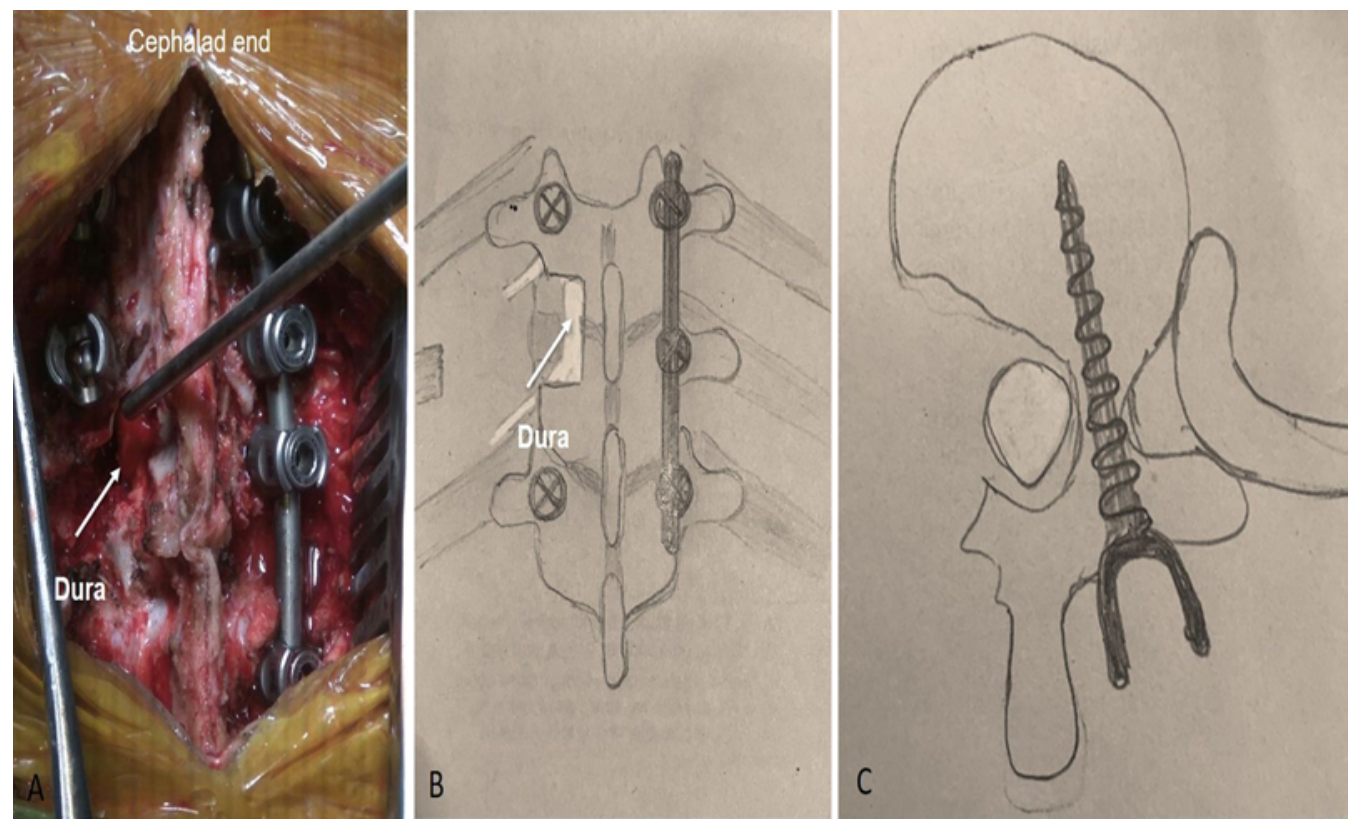

FIG. 3. Cord decompression. Intraoperative photograph (A), illustration from posterior (B), and illustration of the axial cut (C). Figure is available in color online only. 
Yadav et al.

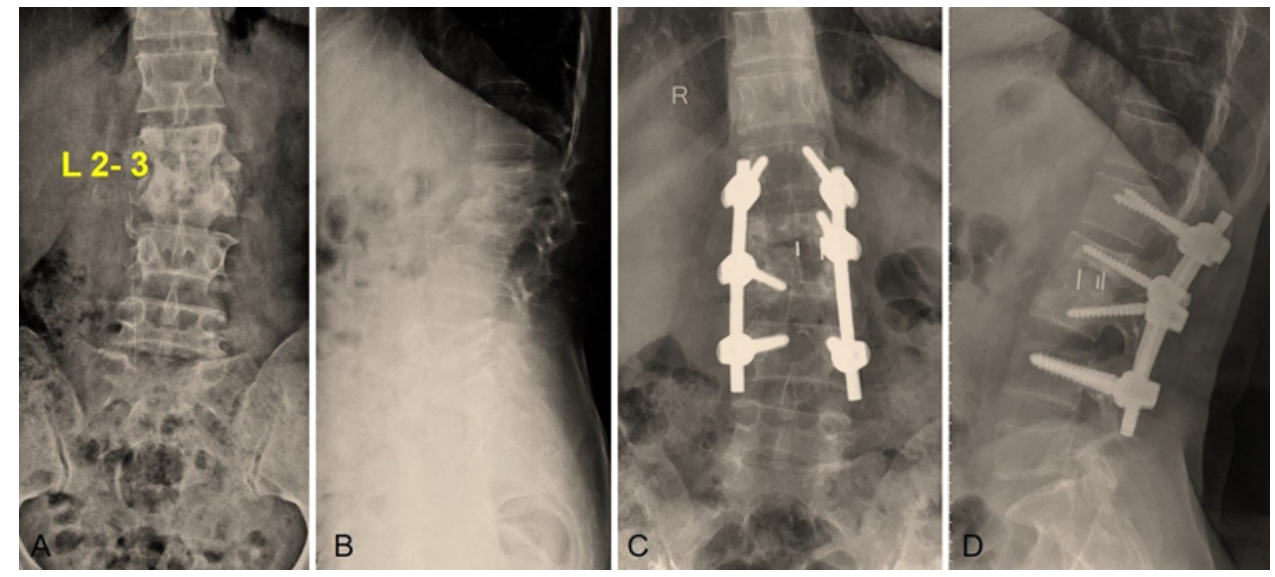

FIG. 5. Case example. Lumbosacral spine radiographs. A 48-year-old woman who had presented with low-back ache for the previous 5 months was given ATT for 6 weeks, but there was no relief in pain; hence, she underwent lamina-sparing decompression and instrumentation. A and B: Preoperative anteroposterior (AP) and lateral views. C and D: Postoperative AP and lateral views. Figure is available in color online only.
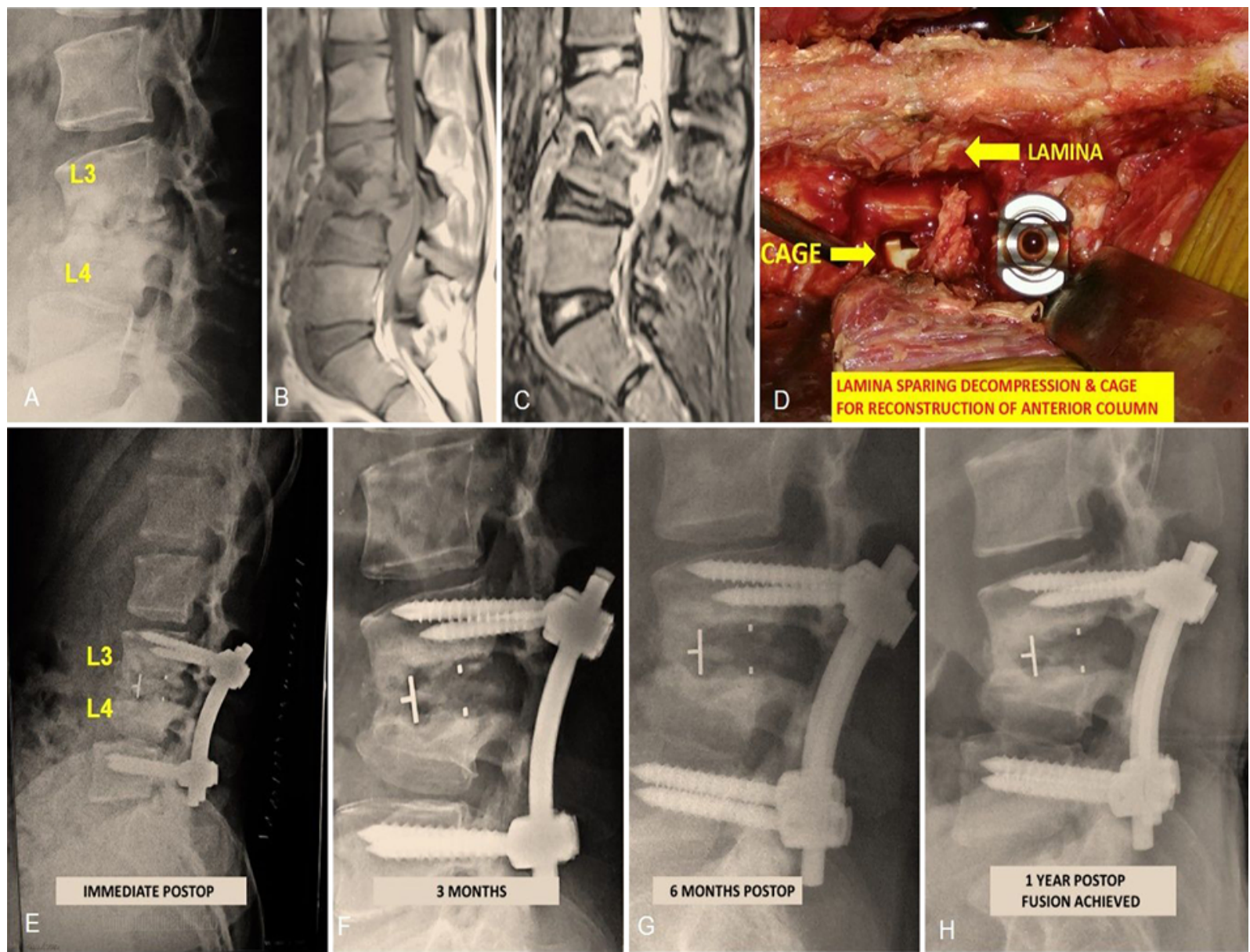

FIG. 6. Case example. Lumbosacral spine radiographs of an 18-year-old woman who presented with low-back pain $(\mathbf{A})$ for 3 months, which was progressive despite intake of ATT for 8 weeks. T1-weighted MRI (B) and STIR (C) images were suggestive of infectious etiology. Lamina-sparing decompression and cage insertion were performed (D). Radiographs performed postoperatively $(\mathbf{E})$ and at 3 months $(\mathbf{F})$ and 6 months $(\mathbf{G})$ show progression to fusion $(\mathbf{H})$, which had been achieved at the 1-year follow-up. Figure is available in color online only. 
cal status showed any deterioration in neurological status after surgery. The average ODI scores were $76.4 \pm 17.9$ (range 32-100) preoperatively, $51.9 \pm 20.2(20-96)$ postoperatively, $14.1 \pm 23.12(0-90)$ at 6 months, and $6.74 \pm$ $17.2(0-60)$ at 16 months. VAS scores were $7.48 \pm 1.16(6-$ 10) preoperatively, $3.74 \pm 1.3(2-6)$ postoperatively, $0.97 \pm$ $2.1(0-8)$ at 6 months, and $0.47 \pm 1.94(0-8)$ at 16 months.

Case examples are shown in Figs. 5-7. Surgical site infections occurred in 2 patients and were managed by wound debridement. A dural tear occurred in 1 patient and was repaired with a Prolene 4-0 suture and fibrin glue. One of the 2 patients who had surgical site infection had implant failure (loosening of the most proximal pedicle screws) (Fig. 8) at follow-up of 3 months. This patient was managed with extension of instrumentation to a more proximal level, and at the time of this report this patient was pain free (VAS score 0/10 and ODI score 6.4\%).

\section{Discussion}

The objective of treating spinal TB is to eradicate the infection, prevent or improve neurological deficits and kyphosis, and achieve spinal stability as well as mobility so that the patient can resume activities of daily living as soon as possible. Patients having neurological deficits or spine instability require debridement and stabilization to achieve these treatment goals. Surgical decompression techniques that can be used are ${ }^{7}$ anterior, anterolateral, or posterolateral decompression, and combined anterolateral and posterolateral decompression and posterior decompression.

We used lamina-sparing decompression with the surgical technique described above (Figs. 1-4). Preoperative kyphosis of $28.4^{\circ} \pm 14.9^{\circ}$ (range $0^{\circ}-60^{\circ}$ ) improved to $16.7^{\circ} \pm 11.3\left(-15^{\circ}\right.$ to $\left.44^{\circ}\right)$ with surgery. Correction of $18.2^{\circ}$ $\pm 12.7^{\circ}\left(0^{\circ}-40^{\circ}\right)$ was achieved, which was statistically significant $(\mathrm{p}<0.5)$. The kyphosis correction achieved in our study is comparable with that reported by $\mathrm{Wu}$ et al. ${ }^{8}$ and $\mathrm{Pu}$ et al., ${ }^{9}$ but less than that obtained by Jain et al., ${ }^{10,12} \mathrm{Garg}$ et al., ${ }^{11}$ Zhang et al., ${ }^{13,14}$ Zeng et al., ${ }^{15}$ Pang et al., ${ }^{16}$ and $\mathrm{Ku}-$ mar et al., ${ }^{17}$ suggesting that good kyphosis correction can be achieved by using lamina-sparing decompression and short-segment instrumentation (Table 1). During followup, our patients overall had loss of kyphosis correction of $1.6^{\circ} \pm 1.5^{\circ}\left(0^{\circ}-5^{\circ}\right)$, which was statistically not significant. Jain et al..$^{10,12}$ in 2 studies had follow-up periods of 1 year, and at the final follow-up in our study the loss of kyphosis correction in our cohort of patients (Table 1) was less than the loss in the patients reported by Jain et al.

The level of instrumentation in our patients was on average $0.97 \pm 0.8$ (range $0-4$ ) vertebra proximal to the proximal most diseased vertebrae and $1.25 \pm 0.75(0-3)$ vertebra distal to the distal most diseased vertebra. Traditionally, instrumentation 2 levels above and 2 levels below the diseased segment is advocated to achieve stability. Most authors, including Lee et al., ${ }^{18}$ Talu et al., ${ }^{19}$ Jain et al., ${ }^{12}$ and Garg et al., ${ }^{11}$ instrumented 2 levels above and 2 levels below the diseased segment, but in our study the same amount of stability was achieved by instrumenting fewer vertebrae. In our cohort, a mean of $2.8 \pm 1.4$ (range 1-6) vertebrae were affected and $3.77 \pm 1.6(0-7)$ verte-

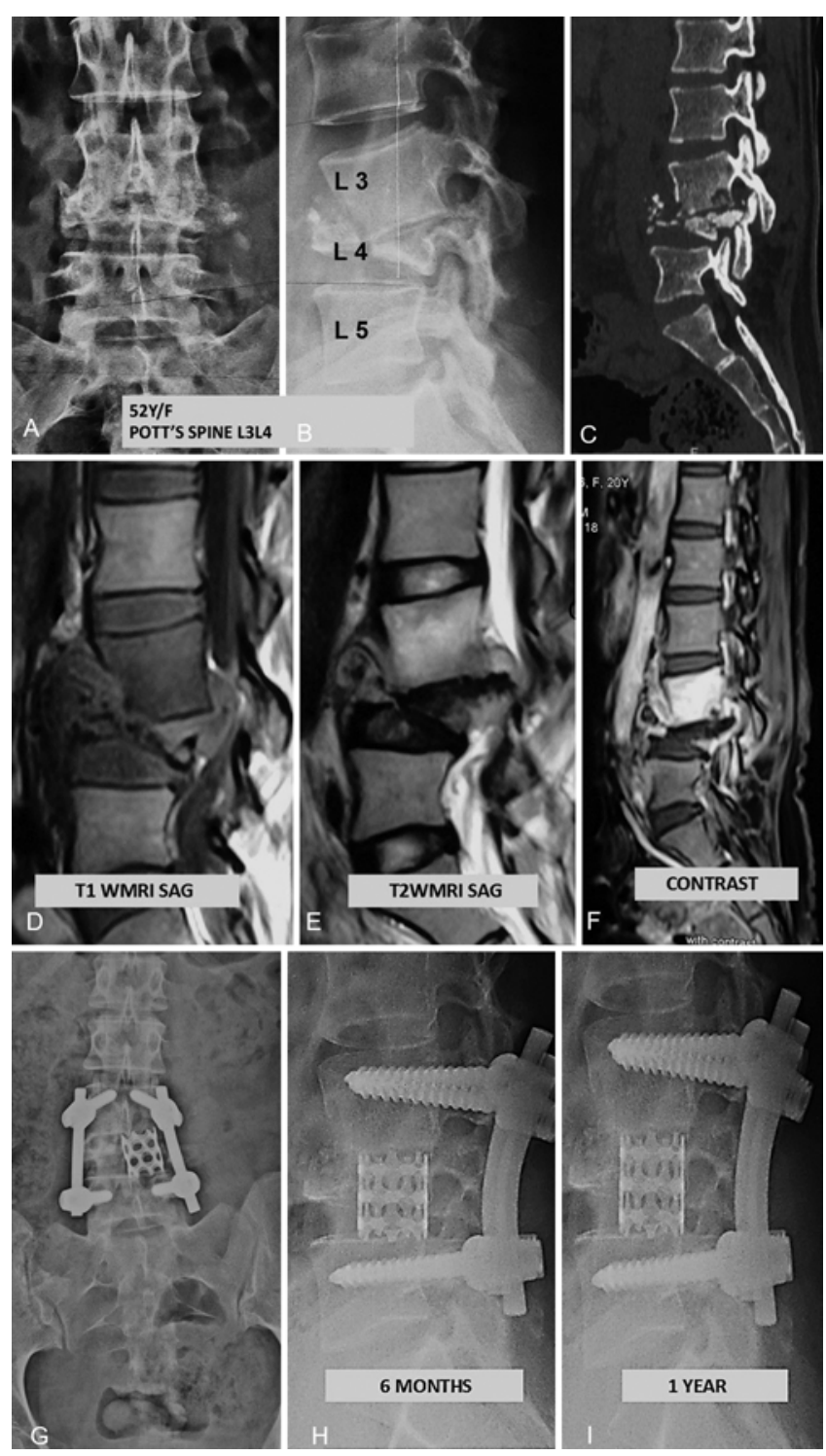

FIG. 7. Case example. A 52-year-old woman who presented with lowback ache for 5 months. Preoperative imaging suggestive of TB spine with complete destruction of $L 4$ and partial destruction of $L 3$ : anteroposterior $(\mathbf{A})$ and lateral $(\mathrm{B})$ radiographs, midsagittal (SAG) CT scan (C), and SAG T1-weighted (T1W; D), T2-weighted (T2W; E), and contrast (F) MRI. Radiographs show lamina-sparing decompression and cage insertion postoperatively $(\mathbf{G})$, at 6 months $(\mathbf{H})$, and at 1 year $(\mathbf{I})$.

brae were instrumented. The lesser level of instrumentation in our method preserved mobility, which was particularly useful in the lumbar spine. Thus, lamina-sparing decompression precludes the need for long instrumentation.

The previously reported incidence of neural complications in spinal TB has been $10 \%$ to $20 \% .{ }^{6}$ Based on the data from government general hospitals in India, nearly $20 \%$ of patients suffering from spinal TB present with neural complications. ${ }^{6}$ In our total cohort of 206 patients, $17 \%$ of patients had neurological deficits and $77.14 \%$ of patients who required surgery had neurological deficits. Out of the total 35 patients in the operative group, 8 had no neurological deficits and 27 did have deficits. Of these 27 

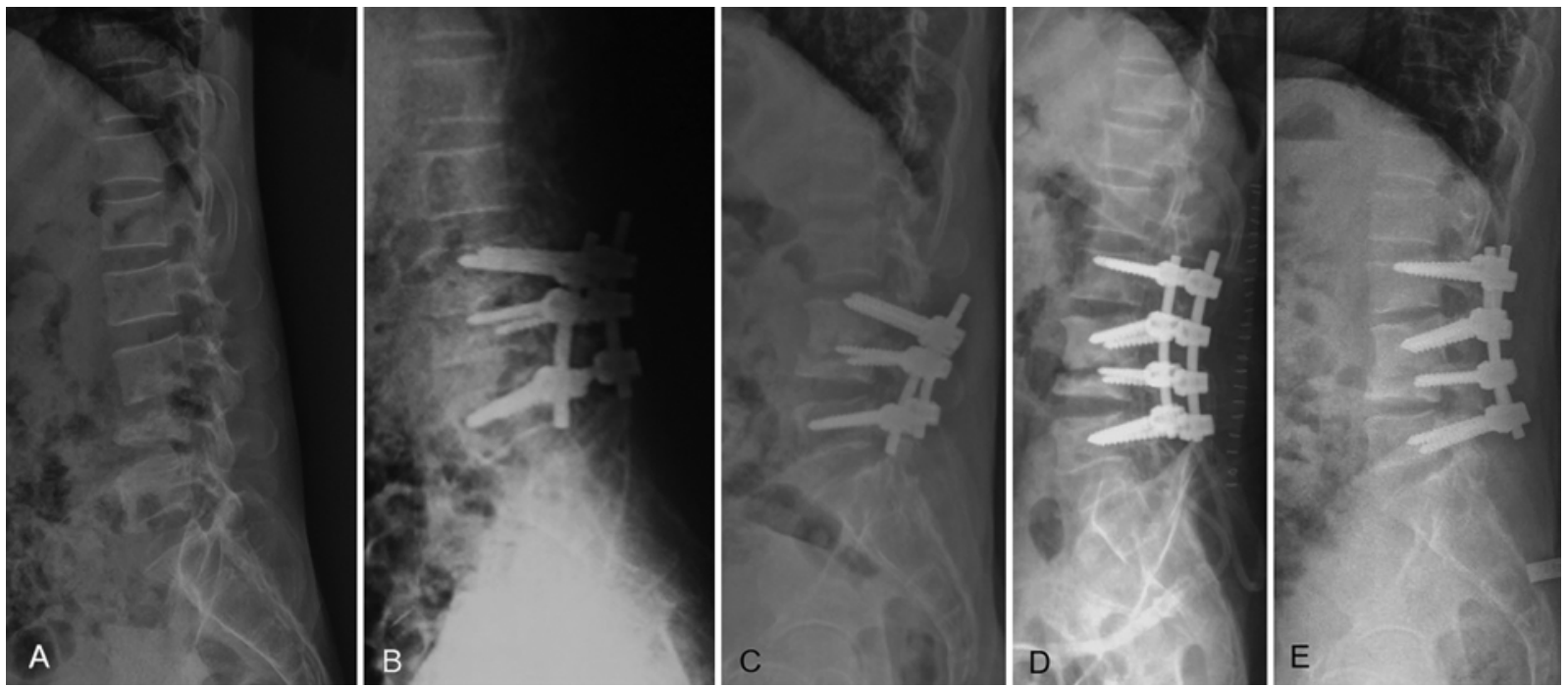

FIG. 8. Case example. Radiographs of a 25-year-old man with TB spondylodiscitis at L3-4 (A) who underwent lamina-sparing decompression and short instrumented fusion from L2 to L4 (B). Implant failure occurred at the 3-month follow-up (C), followed by extension of instrumentation (D). Good bony fusion was seen at the 1-year follow-up (E).

patients, 23 showed improvement during the immediate postoperative period. Overall, $85.1 \%$ of our patients had improvement in neurological status soon after surgery, and all patients improved by the end of 1 year postsurgery. None of the 8 patients with intact neurological status showed any deterioration in neurological status after surgery. Preoperatively, 3 patients had ASIA grade A paraplegia, 3 had ASIA B, 7 had ASIA C, 14 had ASIA grade D, and 8 had ASIA grade E. In the immediate postoperative period, none of the patients had ASIA grade A or B but 2 patients had ASIA grade C, 9 had ASIA grade D, and 24 patients had ASIA grade E. By the end of 1 year postoperatively, none of the patients had ASIA grade A or B, 1 patient had ASIA grade C, 2 patients had ASIA grade $D$, and 32 patients had ASIA grade E. In terms of neurological recovery, the results of our study are comparable to those reported by Zhang et al., ${ }^{14} \mathrm{Wu}$ et al., ${ }^{8}$ Jain et al., ${ }^{10,12} \mathrm{Pu}$ et al., ${ }^{9}$ Zeng et al., ${ }^{15}$ Pang et al., ${ }^{16}$ and Kumar et al. ${ }^{17}$ (Table 1). These results suggest that although lamina-sparing decompression preserves the lamina, adequate decompression is still achieved.

In our cohort of patients, the average VAS scores were

TABLE 1. Patient outcome data for the present study technique compared with previous studies reporting outcome of surgery with conventional techniques in TB spine patients

\begin{tabular}{|c|c|c|c|c|c|c|c|c|}
\hline Authors \& Year & $\begin{array}{l}\text { No. of } \\
\text { Pts }\end{array}$ & $\mathrm{FU}$ (mos) & $\begin{array}{c}\text { Kyphosis } \\
\text { Correction } \\
\text { Achieved (\%) }\end{array}$ & $\begin{array}{l}\text { Kyphosis Loss } \\
\text { at Final FU }\left(^{\circ}\right)\end{array}$ & $\begin{array}{l}\text { Pts w/ Neurologic } \\
\text { Improvement (\%) }\end{array}$ & $\begin{array}{c}\mathrm{p} \\
\text { value }^{*}\end{array}$ & Blood Loss (ml) & Op Duration (mins) \\
\hline Present study & 35 & 12 & $58.1 \pm 15.2 \%$ & $1.6 \pm 1.54(0-5)$ & $100 \%$ & $<0.05$ & $526 \pm 316$ & $228 \pm 79.14(60-360)$ \\
\hline Wu et al., $2013^{8}$ & 21 & $29.8 \pm 5.4$ & $54.5 \%$ & $1 \pm 0.9$ & $100 \%$ & $<0.05$ & $880.2 \pm 112.7$ & $231 \pm 31.9$ \\
\hline Jain et al., $2016^{10}$ & 13 & 12 & $68.5 \%$ & $3.15(1-8)$ & $100 \%$ & $<0.05$ & - & - \\
\hline Pu et al., $2012^{9}$ & 25 & 22.2 & $42.2 \%$ & 6.1 & $100 \%$ & - & 834.1 & 390.2 \\
\hline Jain et al., $2017^{12}$ & 47 & 12 & $65.01 \%$ & $4.1(1-9)$ & $100 \%$ & $<0.05$ & - & - \\
\hline Garg et al., $2012^{11}$ & 36 & 26 & $72.8 \%$ & 2.2 & - & - & 1100 & 290 \\
\hline Zhang et al., $2013^{14}$ & 14 & 31.5 & $63.8 \%$ & 1.3 & $100 \%$ & - & 450 & 110 \\
\hline Zeng et al., $2015^{15}$ & 34 & $34.4 \pm 10.2$ & $76.2 \%$ & $1.5 \pm 0.6$ & $100 \%$ & $<0.05$ & $650.7 \pm 150.2$ & $152.1 \pm 24.4$ \\
\hline Pang et al., $2013^{16}$ & 18 & $34(18-48)$ & $73.6 \%$ & $1-3$ & $100 \%$ & & 815 & $197 \pm 37.9$ \\
\hline Kumar et al., $2013^{17}$ & 25 & 42.6 & $77.8 \%$ & 4.3 & $100 \%$ & - & 667 & 156 \\
\hline Zhang et al., $2012^{13}$ & 60 & $27.5 \pm 3.3$ & $79 \pm 9.9 \%$ & - & $90.1 \%$ & - & 780 & $251 \pm 57.9$ \\
\hline Rawall et al., $2013^{21}$ & 67 & $31.37(12-66)$ & - & - & $89.5 \%$ & - & 514.18 & 239.5 \\
\hline
\end{tabular}

$\mathrm{FU}=$ follow-up; pts $=$ patients.

Values are presented as mean (range) or mean \pm SD (range) unless otherwise indicated.

* Preoperative versus postoperative VAS score. 
$7.48 \pm 1.1$ (range 6-10) preoperatively, $3.74 \pm 1.3(2-6) \mathrm{im}-$ mediately postoperatively, $0.97 \pm 2.1(0-8)$ at 6 months, and $0.47 \pm 1.94(0-8)$ at 16 months. There was a steep decrease in average VAS scores after surgery. VAS score results of our study are comparable to those reported by Wu et al., ${ }^{8}$ Zeng et al. ${ }^{15}$ and Jain et al. ${ }^{12}$ The average ODI scores were $76.4 \pm 17.9$ (range 32-100) preoperatively, $51.9 \pm 20.2$ (20-96) postoperatively, $30.96 \pm 22.05(0-92)$ at 2 months, $14.1 \pm 23.12(0-90)$ at 6 months, and $6.74 \pm 17.2(0-60)$ at 16 months. There was a steep decrease in average ODI scores after surgery.

The average amount of blood loss in our study was 526 \pm 316 (range 130-1200) $\mathrm{ml}$, which is comparatively less than the amounts reported by Wu et al., ${ }^{8} \mathrm{Pu}$ et al., ${ }^{9} \mathrm{Garg}$ et al., ${ }^{11}$ Zeng et al., ${ }^{15}$ Pang et al.. ${ }^{16}$ Kumar et al.. ${ }^{17}$ Zhang et al., ${ }^{13}$ and Jain et al.. ${ }^{20}$ and more than the amounts reported by Zhang et al. ${ }^{14}$ Our results suggest that blood loss in lamina-sparing decompression is less than that occurring with conventional posterior and posterolateral decompression, probably because of minimal removal of healthy posterior elements (Table 1). The average duration of surgery in our study was $228 \pm 79.14$ (range 60-360) minutes, which is comparatively shorter than that reported by Rawall et al., ${ }^{21}$ Pu et al., ${ }^{9}$ Garg et al., ${ }^{11}$ and Jain et al., ${ }^{20}$ but longer than that reported by other researchers, including Kumar et al., ${ }^{17}$ Pang et al.. ${ }^{16}$ and Zeng et al. ${ }^{15}$ (Table 1).

\section{Study Limitations}

The limitations of this study include short follow-up, small sample size, and lack of a control group.

\section{Conclusions}

Lamina-sparing decompression allows the achievement of spinal stability with the instrumentation of fewer vertebrae than are required with other methods. This method leads to good kyphosis correction even with short-segment instrumentation, and loss of kyphosis in postoperative follow-up is also remarkably reduced. This procedure is associated with less blood loss and shorter duration of surgery than other methods because of its retention of as many anatomical structures as possible. Treatment with the lamina-sparing method provides chances of neurological recovery similar to those for treatment with conventional posterior or posterolateral decompression, with good functional improvement after surgery. Hence, lamina-sparing decompression may be a better treatment option than conventional posterior decompression. However, a study with a larger sample size and longer follow-up is needed to confirm these results and assess long-term outcome.

\section{References}

1. Sai Kiran NAS, Vaishya S, Kale SS, et al. Surgical results in patients with tuberculosis of the spine and severe lowerextremity motor deficits: a retrospective study of 48 patients. J Neurosurg Spine. 2007;6(4):320-326.

2. Rasouli MR, Mirkoohi M, Vaccaro AR, et al. Spinal tuberculosis: diagnosis and management. Asian Spine $J$. 2012;6(4):294-308.

3. Ratnappuli A, Collinson S, Gaspar-García E, et al. Pott's disease in twenty-first century London: spinal tuberculosis as a continuing cause of morbidity and mortality. Int J Tuberc Lung Dis. 2015;19(9):1125, i-ii.

4. Rajasekaran S. Kyphotic deformity in spinal tuberculosis and its management. Int Orthop. 2012;36(2):359-365.

5. Kandwal P, G V, Jayaswal A. Management of tuberculous infection of the spine. Asian Spine J. 2016;10(4):792-800.

6. Tuli SM. Historical aspects of Pott's disease (spinal tuberculosis) management. Eur Spine J. 2013;22(Suppl 4):529-538.

7. Jain AK, Dhammi IK, Jain S, Mishra P. Kyphosis in spinal tuberculosis - prevention and correction. Indian J Orthop. 2010;44(2):127-136.

8. Wu P, Luo C, Pang X, et al. Surgical treatment of thoracic spinal tuberculosis with adjacent segments lesion via onestage transpedicular debridement, posterior instrumentation and combined interbody and posterior fusion, a clinical study. Arch Orthop Trauma Surg. 2013;133(10):1341-1350.

9. Pu X, Zhou Q, He Q, et al. A posterior versus anterior surgical approach in combination with debridement, interbody autografting and instrumentation for thoracic and lumbar tuberculosis. Int Orthop. 2012;36(2):307-313.

10. Jain A, Jain R, Kiyawat V. Evaluation of outcome of posterior decompression and instrumented fusion in lumbar and lumbosacral tuberculosis. Clin Orthop Surg. 2016;8(3):268-273.

11. Garg B, Kandwal P, Nagaraja UB, et al. Anterior versus posterior procedure for surgical treatment of thoracolumbar tuberculosis: a retrospective analysis. Indian J Orthop. 2012;46(2):165-170.

12. Jain A, Jain RK, Kiyawat V. Evaluation of outcome of transpedicular decompression and instrumented fusion in thoracic and thoracolumbar tuberculosis. Asian Spine J. 2017;11(1):3136.

13. Zhang H, Huang S, Guo H, et al. A clinical study of internal fixation, debridement and interbody thoracic fusion to treat thoracic tuberculosis via posterior approach only. Int Orthop. 2012;36(2):293-298.

14. Zhang H, Sheng B, Tang M, et al. One-stage surgical treatment for upper thoracic spinal tuberculosis by internal fixation, debridement, and combined interbody and posterior fusion via posterior-only approach. Eur Spine J. 2013;22(3):616-623.

15. Zeng H, Zhang P, Shen X, et al. One-stage posterior-only approach in surgical treatment of single-segment thoracic spinal tuberculosis with neurological deficits in adults: a retrospective study of 34 cases. BMC Musculoskelet Disord. 2015;16:186.

16. Pang X, Shen X, Wu P, et al. Thoracolumbar spinal tuberculosis with psoas abscesses treated by one-stage posterior transforaminal lumbar debridement, interbody fusion, posterior instrumentation, and postural drainage. Arch Orthop Trauma Surg. 2013;133(6):765-772.

17. Kumar MN, Joseph B, Manur R. Isolated posterior instrumentation for selected cases of thoraco-lumbar spinal tuberculosis without anterior instrumentation and without anterior or posterior bone grafting. Eur Spine J. 2013;22(3):624-632.

18. Lee S-H, Sung J-K, Park Y-M. Single-stage transpedicular decompression and posterior instrumentation in treatment of thoracic and thoracolumbar spinal tuberculosis: a retrospective case series. J Spinal Disord Tech. 2006;19(8):595-602.

19. Talu U, Gogus A, Ozturk C, et al. The role of posterior instrumentation and fusion after anterior radical debridement and fusion in the surgical treatment of spinal tuberculosis: experience of 127 cases. J Spinal Disord Tech. 2006;19(8):554-559.

20. Jain AK, Dhammi IK, Jain S, Kumar J. Simultaneously anterior decompression and posterior instrumentation by extrapleural retroperitoneal approach in thoracolumbar lesions. Indian J Orthop. 2010;44(4):409-416.

21. Rawall S, Mohan K, Nene A. Posterior approach in thoracolumbar tuberculosis: a clinical and radiological review of 67 operated cases. Musculoskelet Surg. 2013;97(1):67-75. 


\section{Disclosures}

The authors report no conflict of interest concerning the materials or methods used in this study or the findings specified in this paper.

\section{Author Contributions}

Conception and design: Kandwal, Arora. Acquisition of data: Yadav. Analysis and interpretation of data: Yadav. Drafting the article: Kandwal, Yadav. Critically revising the article: Kandwal, Arora. Approved the final version of the manuscript on behalf of all authors: Kandwal. Statistical analysis: Yadav. Administrative/ technical/material support: Arora. Study supervision: Arora.

\section{Supplemental Information}

\section{Previous Presentations}

Some portions of the contents of the paper were presented at the North American Spine Society 2018 annual meeting in Los Angeles, California, and the Asia Pacific Spine Society 2019 annual meeting in Incheon, South Korea.

\section{Correspondence}

Pankaj Kandwal: All India Institute of Medical Sciences, Uttarakhand, India. pankaj.orth@aiimsrishikesh.edu.in. 